\title{
Tetramethylpyrazine reduces prostate cancer malignancy through inactivation of the DPP10-AS1/CBP/FOXM1 signaling pathway
}

\author{
YI ZHOU $^{1 *}$, ZHIEN ZHOU $^{1 *}$, ZHIGANG JI $^{1}$, WEIGANG YAN ${ }^{1}$, HANZHONG LI $^{1}$ and XIAO YU ${ }^{2}$ \\ Departments of ${ }^{1}$ Urology and ${ }^{2}$ Pathology, Peking Union Medical College Hospital, \\ Chinese Academy of Medical Science and Peking Union Medical College, Beijing 100000, P.R. China
}

Received August 31, 2019; Accepted February 6, 2020

DOI: $10.3892 /$ ijo.2020.5036

\begin{abstract}
Tetramethylpyrazine (TMP), a Chinese herbal medicine, has been reported to possess anticancer effects. Emerging evidence suggests that various long noncoding RNAs (lncRNAs) serve important roles in cancer initiation and progression. In the present study, the tumor-suppressive effects of TMP in human PCa cells was examined and the underlying mechanisms of its actions were determined. The data showed that TMP treatment reduced cell viability and increased apoptosis in a dose-dependent manner. Reverse transcription-quantitative PCR showed TMP treatment increased the expression of 1ncRNA DPP10-AS1 in PCa cells. Furthermore, DPP10-AS1 was also upregulated in TMP-resistant PCa cells. Knockdown of DPP10-AS1 reversed TMP resistance, whereas increased expression of DPP10-AS1 abrogated the TMP-mediated cytotoxicity in PCa cells. In addition, forkhead box M1 (FOXM1) was verified as the functional target of DPP10-AS1, and knockdown of FOXM1 reversed the TMP/DPP10-AS1-induced cell cytotoxicity. Mechanistically, DPP10-AS1 was associated with CREB binding protein, thereby induced $\mathrm{H} 3 \mathrm{~K} 27 \mathrm{ac}$ enrichment at the promoter region of the FOXM1 gene. In conclusion, the present study showed that TMP may be a promising treatment agent for $\mathrm{PCa}$ and lncRNA DPP10-AS1 may be a promising therapeutic target for TMP treatment.
\end{abstract}

\section{Introduction}

Prostate cancer ( $\mathrm{PCa}$ ) is one of the most common types of cancer, and is a leading cause of cancer-associated among men

Correspondence to: Dr Weigang Yan, Department of Urology, Peking Union Medical College Hospital, Chinese Academy of Medical Science and Peking Union Medical College, 1 Shuaifuyuan, Wangfujing Road, Dongcheng, Beijing 100730, P.R. China

E-mail: bjyanweig@163.com

*Contributed equally

Key words: prostate cancer, tetramethylpyrazine, DPP10-AS1, forkhead box M1, cell cytotoxicity worldwide (1). Tumor growth in the early-stages of prostate cancer development is dependent on the presence of androgens, and androgen deprivation therapy (ADT) is the primary treatment used for androgen-dependent prostate cancer (2). However, a notable subset of patients experience recurrence and progression following the failure of ADT, which is the leading cause of PCa-associated death (3). Therefore, understanding the pathogenesis of PCa and identifying novel treatment choices are urgently required.

Tetramethylpyrazine (TMP) is one of the active compounds extracted from the Chinese medicinal plant Ligusticum chuanxiong (4). TMP has been widely used in Chinese herbal medicines for various purposes, including treating neurovascular and cardiovascular diseases, as it possesses anti-oxidant and anti-inflammatory properties (5). TMP has been shown to influence cancer progression in lung cancer (6), bladder cancer (7), breast cancer (8) and gastric cancer (9), beyond its traditional roles. In our previous study, it was shown that TMP exerted anti-cancer effects in PCa (10). To the best of our knowledge, there are no studies examining the molecular mechanisms underlying the effects of TMP in cancer.

Long-chain non-coding RNA (lncRNA) is a type of non-coding RNA, 200-100,000 bases in length, which lack an effective open reading framework and is transcribed by RNA polymerase II without protein coding function (11). LncRNAs serve as transcription regulators through interfering with the binding of transcription factors to promoters, interfering with gene transcription and chromatin remodeling; or by binding to proteins through chaperones, regulating subcellular localization of proteins (12-14). The antisense lncRNAs are a subtype of lncRNA molecules transcribed from the opposite DNA strand of protein-coding or non-protein-coding genes (15). At present, only a few antisense lncRNAs have been extensively studied, such as AGAP2-AS1 (16) and ZEB1-AS1 (17). Whether antisense lncRNAs participate in the progression of $\mathrm{PCa}$ and the TMP-induced anti-cancer effects, and the mechanisms underlying the regulatory mechanisms of TMP have not been studied.

In the present study, the antisense lncRNA termed DPP10-AS1, which is located on chr 2q14.1, and contains 744 nt was examined in PCa. The status of DPP10-AS1 in PCa has not been investigated to the best of our knowledge, thus, the expression levels and functional roles of DPP10-AS1 of 
DPP10-AS1 in PCa, and its role in TMP-induced anti-cancer effects were determined. It was shown that DPP10-AS1 expression was downregulated in TMP-treated PCa cells, and upregulation of DPP10-AS1 reversed the anti-cancer effects induced by TMP. Mechanistically, DPP10-AS1 increased the expression of forkhead box M1 (FOXM1) via H3K27 acetylation modification at the FOXM1 promoter region.

\section{Materials and methods}

Clinical specimen. A total of 35 pairs of PCa tissues and adjacent normal tissues were collected by puncture biopsy between January 2014 and June 2017, and snap-frozen in liquid nitrogen instantly. The median age of the patients recruited in the present study was 53 years (age range, 42-69 years). Patients who received radiotherapy and chemotherapy prior to puncture biopsy were excluded. The enrolled patients provided written informed consent, and the present study was approved by the Research Ethics Committee of Peking Union Medical College Hospital.

Cell culture and treatment. The PCa cell lines, PC3 and DU145, and one prostate epithelial cell line, hPrEC, were all purchased from Shanghai Institutes for Biological Sciences Cell Resource Center (Shanghai, China). PC3 and DU145 cells were grown in DMEM (Thermo Fisher Scientific, Inc.) supplemented with glucose and 10\% FBS (Thermo Fisher Scientific, Inc.). hPrEC cells were cultured in prostate epithelium basal media (Shanghai Bangjing Industry Co., Ltd.) and all the cells were cultured in a humidified incubator at $37^{\circ} \mathrm{C}$ with $5 \% \mathrm{CO}_{2}$. The TMP-resistant PC3 sub-line (PC3-R) was established by continuously treating PC3 cells with TMP (500 $\mu \mathrm{g} / \mathrm{l}$; Mansite Biotechnology, Co., Ltd.) for 3 months.

Cell transfection. Small interfering (si)RNAs targeting DPP10-AS1 (si-DPP10-AS1, GFP-labeled) and FOXM1 (si-FOXM1) plasmids were synthesized by Shanghai GenePharma Co., Ltd. DPP10-AS1, CREB binding protein (CBP) and FOXM1 cDNA was generated and subcloned into a pcDNA3.1 vector, termed p-DPP10-AS1, p-CBP or p-FOXM1, respectively. p-DPP10-AS1 was further cloned into an Ad.Max ${ }^{\mathrm{TM}}$ adenovirus vector (Shanghai GeneChem Co., Ltd.) for in vivo studies. Plasmid constructs were transfected into cells at 70-90\% confluency using Lipofectamine ${ }^{\circledR} 2000$ (Invitrogen; Thermo Fisher Scientific, Inc.) for $6 \mathrm{~h}$ with a final concentration of $100 \mathrm{nM}$ plasmid according to the manufacturer's protocol. The sequences of the siRNAs are presented in Table I.

RNA extraction and reverse transcription-quantitative $(R T-q) P C R$. Total RNA was extracted using TRIzol ${ }^{\circledR}$ reagent (Thermo Fisher Scientific, Inc.). The concentration of extracted RNA was determined using a Qubit 4.0 spectrophotometer (Thermo Fisher Scientific, Inc.). For DPP10-AS1 and FOXM1 expression analysis, RT was performed using a TaqMan High-Capacity cDNA Reverse Transcription kit and a TaqMan Fast PCR Master mix (Applied Biosystems; Thermo Fisher Scientific, Inc.) according to the manufacturer's protocol. The thermocycling conditions for qPCR were: $95^{\circ} \mathrm{C}$ for $30 \mathrm{sec}$; followed by 45 cycles of $95^{\circ} \mathrm{C}$ for $5 \mathrm{sec}$ and $60^{\circ} \mathrm{C}$ for $30 \mathrm{sec}$. At the beginning of the logarithmic phase of PCR amplification, cycle quantifications $(\mathrm{Cq})$ were appointed and the repeated $\mathrm{Cq}$ values were analyzed using the $2^{-\Delta \Delta \mathrm{cq}}$ method (18). GAPDH was used to normalize the relative expression levels of lncRNA and mRNA. The sequences of the primers are shown in Table I.

Cell viability assay. PCa cells $\left(1 \times 10^{5}\right.$ cells per well) were seeded in 96-well plates and cultured for $24 \mathrm{~h}$ prior to analysis of cell proliferation using a Cell Counting Kit-8 (CCK-8) assay (Dojindo Molecular Technologies, Inc.). Cells were cultured for a further 24,48 or $72 \mathrm{~h}$. After the set incubation times, $10 \mu \mathrm{l}$ CCK-8 solution was added and cells were further incubated at $37^{\circ} \mathrm{C}$ for $4 \mathrm{~h}$. To obtain cell growth curves, the optical density of the plates were measured at $450 \mathrm{~nm}$ using a microplate spectrophotometer (Thermo Fisher Scientific, Inc.). All experiments were performed in triplicate.

TUNEL assay. An in-situ cell death detection kit (Roche Diagnostics) was used to measure cell apoptosis according to the manufacturer's protocol. Transfected cells $\left(5 \times 10^{5}\right.$ cells/well) were cultured in a 24 -well plate for $24 \mathrm{~h}$. Subsequently, cells were fixed with $4 \%$ paraformaldehyde for $60 \mathrm{~min}$ at room temperature and subsequently incubated in $0.1 \%$ Triton X-100 for $2 \mathrm{~min}$ on ice. After permeabilization with Triton X-100, a total of $50 \mu \mathrm{l}$ mixed TUNEL solution (prepared according to the manufacturer's protocol) was added and cells were incubated at $37^{\circ} \mathrm{C}$ for $1 \mathrm{~h}$. The nuclei of cells were stained using DAPI for $1 \mathrm{~h}$ at room temperature and images were taken using a fluorescence microscope (magnification, x20; Nikon Corporation).

Bioinformatics analysis. The putative binding site of H3K27ac at the promoter region of DPP10-AS1 gene was predicted using UCSC Genome Browser (19). The full sequence of DPP10-AS1 gene can be accessed with the NCBI Reference Sequence: NC_00002.12.

RNA immunoprecipitation (RIP) and chromatin immunoprecipitation (ChIP). RIP analysis was performed in $\mathrm{PCa}$ cells using a Magna RIP RNA-binding protein immunoprecipitation kit (EMD Millipore) according to manufacturer's protocol. Briefly, cells were collected after washing with cold PBS and RIP lysis buffer was added. The suspension was then centrifuged and $100 \mu \mathrm{l}$ from each cell lysate was transferred to the RIP immunoprecipitation buffer, which contained CBP antibody (1:100; Abcam; cat. no. ab2832) or IgG as the negative control (1:200; EMD Millipore; cat. no. 12-371). The magnetic beads were washed with RIP wash buffer and incubated with proteinase $\mathrm{K}$ at $55^{\circ} \mathrm{C}$ for $30 \mathrm{~min}$. Subsequently, RNA was extracted for RT-qPCR analysis.

ChIP assay was performed using an EZ-Magna ChIP kit (EMD Millipore). To generate the chromatin fragments (200-300 bp in length) cells were treated with formaldehyde for $10 \mathrm{~min}$ followed by sonication at $4^{\circ} \mathrm{C}$. Subsequently, ChIP-specific antibodies against H3K27ac (1:100; Abcam; cat. no. ab4729), CBP (1:100; Abcam; cat. no. ab2832) and normal mouse IgG polyclonal antibody (1:100; EMD Millipore; cat. no. 12-371) were used for immunoprecipitation. RNA was recovered using an EpiTect ChIP qPCR assays (cat. no. 334001; Qiagen, Inc.), according to the manufacturer's protocol and analyzed by qPCR. 
Table I. Sequences of the primers and siRNAs.

\begin{tabular}{ll}
\hline Gene & \multicolumn{1}{c}{ Sequence, 5'-3' } \\
$\begin{array}{l}\text { DPP10-AS1 } \\
\text { Forward } \\
\text { Reverse }\end{array}$ & AGATTGTGGCCTGAGGTGC \\
FOXM1 & TTAGGAGTTCCACCGACGTG \\
Forward & GGAGGAAATGCCACACTTAGCG \\
Reverse & TAGGACTTCTTGGGTCTTGGGGT \\
CREB binding & \\
protein & \\
Forward & \\
Reverse & GTGCTGGCTGAGACCCTAAC \\
GAPDH & GGCTGTCCAAATGGACTTGT \\
Forward & \\
Reverse & GCACCGTCAAGGCTGAGAAC \\
si-DPP10-AS1 & ATGGTGGTGAAGACGCCAGT \\
si-FOXM1 & CCUAAAGGGAUGCCUUCAATT \\
si-negative & UAGUAACUCUGGCCAUAGCTT \\
control & CAGGUGGACUCACAAUUCCTT \\
\hline
\end{tabular}

siRNA, small interfering; FOXM1, forkhead box M1.

Nucleocytoplasmic separation. Nuclear and cytosolic fractions were separated using a Nuclear/Cytosol Fractionation kit (cat. no. K266-25; BioVision, Inc.) according to the manufacturer's protocol. Subsequently, RT-qPCR was used to determine the expression of DPP10-AS1, GAPDH and U1 in the nucleus and cytoplasm of PC3 and PC3-R cells.

RNA florescent in situ hybridization (FISH). Hybridization was performed using a FISH kit (Guangzhou RiboBio Co., Ltd.) according to the manufacturer's protocol. Briefly, $4 \%$ paraformaldehyde was used to fix the PCa cells (15 min at room temperature) followed by permeabilization with $0.5 \%$ Triton X-100 for $15 \mathrm{~min}$ at room temperature. Subsequently, the cells were cultured with specific GFP-labeled DPP10-AS1 probes (Guangzhou RiboBio Co., Ltd.) overnight at $4^{\circ} \mathrm{C}$. All fluorescent images were captured using Nikon A1Si Laser Scanning Confocal Microscope (magnification x100; Nikon Corporation).

Immunohistochemistry (IHC) analysis and scoring methods. Tumor tissues from nude mice were rehydrated using a graded sequence of ethanol solutions (70, 85, 95 and 100\%) followed by deionized water. Subsequently, tissues were immersed in citrate buffer $(0.01 \mathrm{~mol} / \mathrm{l})$ and heated to $95^{\circ} \mathrm{C}$ for $30 \mathrm{~min}$. Slides were washed with PBS solution followed by treatment with $1 \%$ Triton X-100 solution for $30 \mathrm{~min}$ at room temperature, and stained using a biotin-streptavidin CytoScan ${ }^{\mathrm{TM}}$ horseradish peroxidase Detection system according to the manufacturer's protocol (EMD Millipore), followed by incubation with a primary antibody targeting FOXM1 (1:200; Abcam; cat. no. ab184637) overnight at $4^{\circ} \mathrm{C}$. The presence of brown chromogen in the cytoplasm indicated positive immunoreactivity.

The immunostaining intensity of each sample was graded as follows: Negative, 0; weak, 1; moderate, 2; or strong, 3.
The proportion of positively stained cells is represented as a percentage of the total number of cells. The final score was then calculated as the intensity score multiplied by the percentage of cells stained. Images were visualized using a confocal Nikon ECLIPSE Ti (magnification x40; Nikon Corporation) and processed using Nikon NIS-Elements software (version 1.0; Nikon Corporation).

In vivo nude mouse model. Tumor xenografts were established using male BALB/c nude mice (4-6 weeks old), which were purchased from the Model Animal Research Center of Nanjing University. PC3 cells were stably infected with p-DPP10-AS1 or p-NC, and $3 \times 10^{6}$ cells were subcutaneously injected into nude mice followed by treatment with TMP or PBS $(50 \mathrm{mg} / \mathrm{kg}$ ) once every two days for 6 weeks. The mice were divided into 3 groups (5 mice in each group): i) Group I, PBS treatment + p-NC; ii) group II, TMP treatment + p-NC; and iii) group III, TMP treatment + p-DPP10-AS1. Subsequently the mice were sacrificed after 30 days, the grafted tumors were removed, and the weights of the neoplasms were measured immediately after resection. After weighing, the tissues were fixed for sectioning with $4 \%$ paraformaldehyde followed by IHC staining for FOXM1, as described above. Animal experiments were approved by the Institutional Review Board of Peking Union Medical College Hospital.

Western blotting. RIPA lysis buffer consisting of $150 \mathrm{mM}$ $\mathrm{NaCl}, 1 \%$ Triton X-100, $0.5 \%$ sodium deoxycholate, $0.1 \%$ SDS, $50 \mathrm{mM}$ Tris $(\mathrm{pH} 8)$ and protease inhibitors cocktail (Promega Corporation) was used to lyse the cells and exosome samples. Protein concentration was measured using a bicinchoninic acid assay (cat. no. BCA1-1KT, Sigma Aldrich; Merck KGaA). Proteins (25 mg per lane) were loaded on a $10 \%$ SDS gel, resolved using SDS-PAGE and transferred to PVDF membranes using a Trans-Blot system (Bio-Rad Laboratories, Inc.). Subsequently, the membranes were incubated with specific primary antibodies against FOXM1 (1:1,000; Abcam; cat. no. ab184637), CBP (1:1,000; Abcam; cat. no. ab2832), and GAPDH (1:5,000; Abcam; cat. no. ab9485) at $4^{\circ} \mathrm{C}$ overnight and subsequently incubated with the goat anti-rabbit polyclonal horseradish peroxidase-conjugated secondary antibody (1:5,000; Abcam; cat. no. ab7090) for $1 \mathrm{~h}$ at room temperature. Membranes were developed using an enhanced chemiluminescent reagent and visualized using a ChemiDoc XRS Imaging system and analyzed using the accompanying software Image Lab version 3.0 (Bio-Rad Laboratories, Inc.).

Statistical analysis. GraphPad Prism Software version 5.0.1 (GraphPad Software, Inc.) was used to perform statistical analysis. A two-tailed Student's t-test, one-way ANOVA with a Tukey's post-hoc test or Pearson's correlation analysis were used to compare differences. Data are presented as the mean \pm standard error of the mean. $\mathrm{P}<0.05$ was considered to indicate a statistically significant difference.

\section{Results}

TMP reduces the proliferation and promotes apoptosis of PCa cells by targeting DPP10-AS1. Our previous study showed that PC3 and DU145 are appropriate cell models 

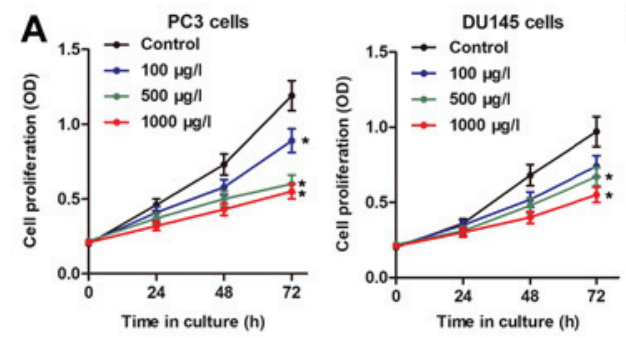

$\mathbf{B}$
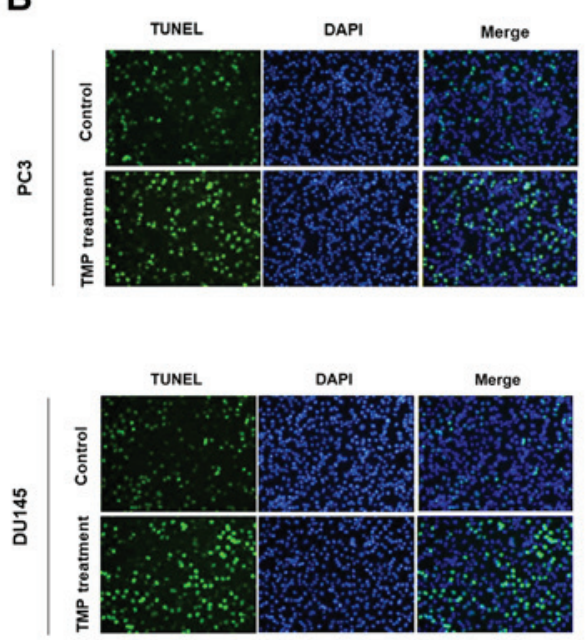

C

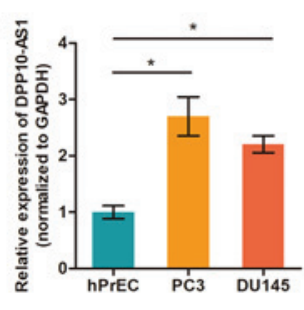

D

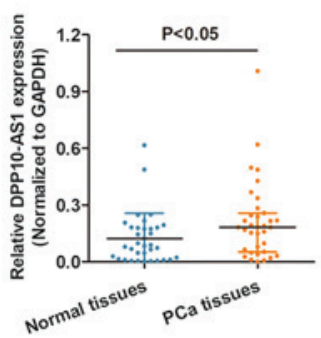

E

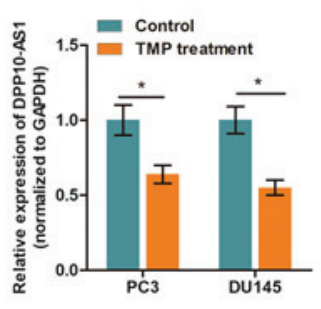

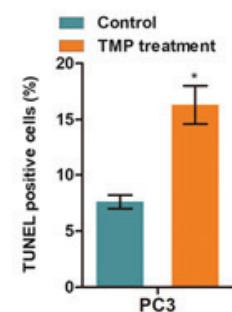

F

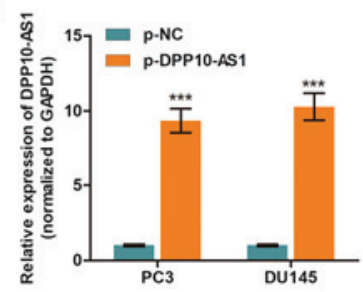

H

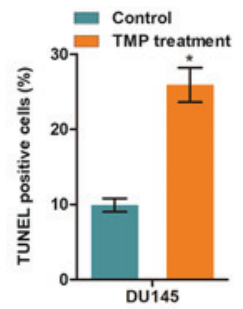

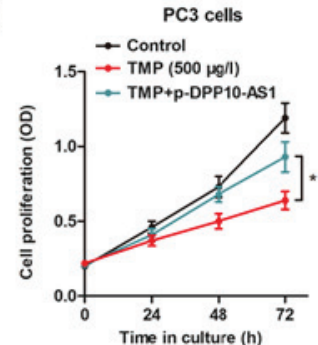
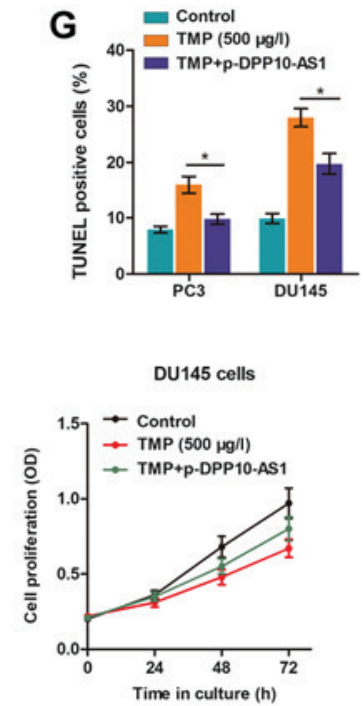

Figure 1. DPP10-AS1 is involved in the TMP-induced anti-PCa effects. (A) A CCK-8 assay showed that cell viability was decreased in PCa cells treated with TMP in a concentration-dependent manner. " $\mathrm{P}<0.05$ vs. Control. (B) A TUNEL assay showed that TMP treatment increased apoptosis. "P<0.05. (C) RT-qPCR analysis showed that DPP10-AS1 expression was upregulated in PC3 and DU145 compared with normal prostate epithelial hPrEC cells. "P<0.05. (D) RT-qPCR analysis showed that DPP10-AS1 expression was significantly upregulated in PCa tissues compared with the adjacent normal tissues. (E) RT-qPCR analysis showed that $500 \mu \mathrm{g} / 1$ TMP treatment significantly decreased DPP10-AS1 expression in PC3 and DU145 cells. "P<0.05. (F) DPP10-AS1 expression was significantly increased following transient transfection of a DPP10-AS1 overexpression vector in PCa cells. ${ }^{* * *} \mathrm{P}<0.001$. (G) A TUNEL assay confirmed that DPP10-AS1 overexpression partially reversed TMP-induced cell apoptosis. ${ }^{*} \mathrm{P}<0.05$. (H) A CCK-8 assay showed that overexpression of DPP10-AS1 partially reversed TMP-induced tumor suppression effects. " $\mathrm{P}<0.05$. TMP, tetramethylpyrazine; PCa, prostate cancer; CCK-8, Cell Counting Kit-8; RT-qPCR, reverse transcription-quantitative PCR; OD, optical density; NC, negative control; p-, pcDNA3.1 overexpression vector.

for assessing the effects of TMP in PCa (10). To confirm the anti-cancer effects of TMP in PCa, the cell viability of cells treated with different concentrations of TMP was determined. The CCK-8 assay showed that cell viability was suppressed in a concentration-dependent manner (Fig. 1A), whereas the TUNEL assay showed that TMP increased cell death (Fig. 1B), suggesting that TMP reduced progression of PCa. To determine whether DPP10-AS1 was involved in the TMP-induced anti-cancer effects, the expression levels of DPP10-AS1 in PCa were measured. Fig. 1C showed that DPP10-AS1 expression was upregulated in PC3 and DU145 cells compared with prostate epithelial cells. In addition, DPP10-AS1 levels were also increased in PCa tissues compared with normal tissues (Fig. 1D). Importantly, TMP treatment significantly reduced DPP10-AS1 levels in PCa cells compared with the control cells (Fig. 1E). To verify the functional role of DPP10-AS1, DPP10-AS1 was overexpressed in PCa cells treated with TMP (Fig. 1F), and it was shown that DPP10-AS1 significantly reversed the anti-cancer effects of TMP in PCa cells, as evidenced by increase in apoptosis and proliferation (Fig. 1G and $\mathrm{H}$ ).

Overexpression of DPP10-AS1 reverses TMP resistance in PCa cells. To further verify the role of DPP10-AS1 in
TMP-induced anti-tumor effects, a TMP-resistant PC3 sub-line (PC3-R) was established by continuously treating PCa cells with TMP $(500 \mu \mathrm{g} / \mathrm{l})$ for 3 months. As shown in Fig. 2A, PC3-R cells showed specific morphological changes in contrast to the sensitive parental cell lines, such as reduced cell polarity, increased number of pseudopodia and enlarged intercellular separation. Furthermore, the established PC3-R cells showed increased cell viability compared with the parental cells when treated with $500 \mathrm{mg} / 1$ TMP (Fig. 2B and C). RT-qPCR analysis showed expression of DPP10-AS1 was significantly higher in the PC3-R cells compared with the PC3 cells (Fig. 2D). Therefore, DPP10-AS1 expression was downregulated in PC3-R cells by transient transfection of specific siRNA (Fig. 2E and F). As shown in Fig. 2F, knockdown of DPP10-AS1 partially reversed TMP resistance of PC3-R cells as evidenced by increased cell cytotoxicity caused by TMP treatment. Together, these data suggest that DPP10-AS1 is essential for TMP resistance in PCa cells.

LncRNA DPP10-AS1 mediates TMP-induced cell cytotoxicity by directly targeting FOXM1. To identify the target genes of DPP10-AS1, StarBase v2.0 was used (20), and 7 candidate genes associated with DPP10-AS1 were identified (Table II). In addition, in our previous study it was shown that TMP 
A

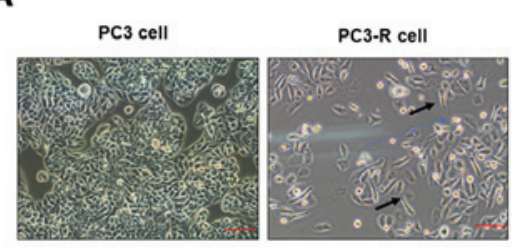

B

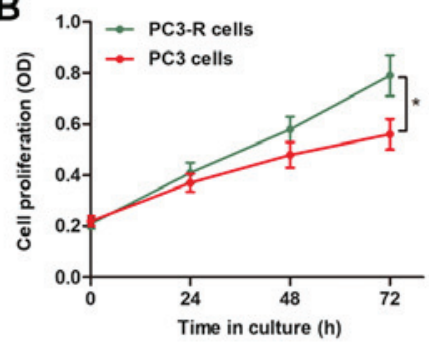

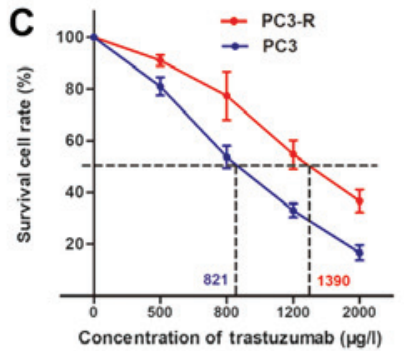

G

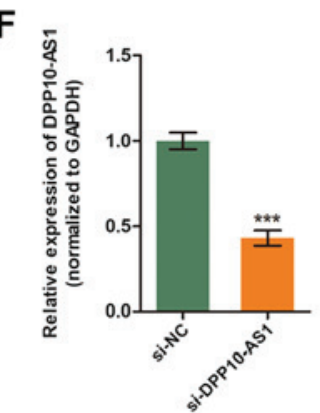

G

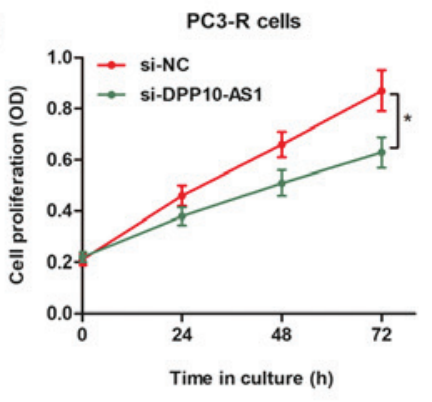

E

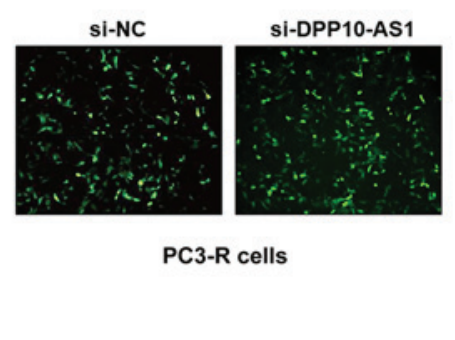

Figure 2. Overexpression of DPP10-AS1 reverses TMP resistance in PCa cells. (A) Morphological presentation of the TMP resistant PC3-R cells and the parental PC3 cells. PC3-R cells showed specific changes compared with parental cells, including loss of cell polarity, increased formation of pseudopodia and intercellular separation. (B) Cell Counting Kit-8 assay showed that PC3-R cells exhibited increased cell viability compared with PC3 cells when treated with $500 \mu \mathrm{g} / 1$ TMP. ${ }^{*} \mathrm{P}<0.05$. (C) PC3-R cells showed exhibited higher $\mathrm{IC}_{50}$ values compared with the PC3 cells (1,390 vs. $821 \mathrm{mg} / 1$, respectively). (D) A reverse transcription-quantitative PCR assay showed that DPP10-AS1 expression was upregulated in PC3-R cells compared with the PC3 cells. * $<0.05$. (E) GFP-labelled siRNAs targeting DPP10-AS1 was transfected into PC3-R cells. (F) Expression of DPP10-AS1 was knocked down by transfection of si-DPP10-AS1 in PC3-R cells. ${ }^{* * *} \mathrm{P}<0.001$. (G) Knockdown of DPP10-AS1 increased TMP-induced cell cytotoxicity in PC3-R cells. * $\mathrm{P}<0.05$. TMP, tetramethylpyrazine; si, small interfering; OD, optical density; NC, negative control.

inhibited PCa progression by downregulating the expression of FOXM1 (10). Thus, the expression levels of these 8 potential target genes was assessed. The results showed that the expression of FOXM1 was upregulated by DPP10-AS1 overexpression and downregulated when DPP10-AS1 was silenced (Fig. 3A). Furthermore, FOXM1 expression levels were higher in the TMP-resistant PC3-R cells compared with the PC3 cells (Fig. 3B). The other 7 genes were not regulated by DPP10-AS1 (data not shown). To determine whether FOXM1 was a functional target of DPP10-AS1, FOXM1 knockdown and overexpression vectors were transfected into the PC3 and PC3-R cells, respectively (Fig. 3C). By performing a CCK8 assay, it was shown that silencing of FOXM1 abrogated the influence of DPP10-AS1 in PC3 cells treated with TMP (Fig. 3D). Similarly, overexpression of FOXM1 partially reversed DPP10-AS1 knockdown-induced effects in PC3-R cells (Fig. 3E).

LncRNA DPP10-AS1 increases FOXM1 expression by inducing $H 3 K 27$ acetylation at the promoter region. It has been shown that the CBP, an acetyltransferase, is recruited to the C-terminal region of FOXM1 and enhances its transcriptional activity at specific stages of the cell cycle (21). To verify whether DPP10-AS1 upregulates FOXM1 by histone acetylation, mediated by $\mathrm{H} 3 \mathrm{~K} 27 \mathrm{ac}$ modification at the C-terminal region, the transcriptional modification regions were analyzed using UCSC Genome Browser. Fig. 4A showed the presence of a potential region which may be enriched in $\mathrm{H} 3 \mathrm{~K} 27 \mathrm{ac}$ upstream of the FOXM1 promoter region. Subsequently, ChIP was performed using an anti-H3K27ac antibody in PCa cells. The results showed that $\mathrm{H} 3 \mathrm{~K} 27 \mathrm{ac}$ was enriched at the
FOXM1 promoter region in both PC3 and PC3-R cells. In addition, the enrichment level of $\mathrm{H} 3 \mathrm{~K} 27 \mathrm{ac}$ was significantly increased in PC3-R cells compared with PC3 cells (Fig. 4B). To further determine whether DPP10-AS1 regulated H3K27ac enrichment, the subcellular localization of DPP10-AS1 was determined in PCa cells. RT-qPCR analysis of DPP10-AS1 in the nucleus and cytoplasm showed that DPP10-AS1 was primarily distributed in the nucleus (Fig. 4C). The results of a FISH assay with a DPP10-AS1 specific probe showed results consistent with the RT-qPCR results (Fig. 4D), suggesting that DPP10-AS1 was primarily located in the nucleus of PCa cells. Overexpression of DPP10-AS1 significantly increased the enrichment of H3K27ac, whereas knockdown of DPP10-AS1 decreased the enrichment levels (Fig. 4E). Furthermore, TMP treatment $(500 \mu \mathrm{g} / \mathrm{l})$ decreased $\mathrm{H} 3 \mathrm{~K} 27 \mathrm{ac}$ enrichment at the FOXM1 promoter region in PC3 cells (Fig. 4F). Together, these results show that DPP10-AS1 may increase FOXM1 expression by increasing $\mathrm{H} 3 \mathrm{~K} 27 \mathrm{ac}$ modifications at the promoter region of FOXM1.

CBP is essential for DPP10-AS1-mediated H3K27ac modifications. CBP is a histone acetyltransferase which serves an important role in histone acetylation (22). To determine whether CBP participated in DPP10-AS1-induced histone modification, CBP overexpression plasmids were transfected into the PC3 and PC3-R cells (Fig. 5A). As shown in Fig. 5B, overexpression of CBP promoted FOXM1 expression at both the mRNA and protein expression levels. Thus whether DPP10-AS1 directly interacted with CBP was determined and the results showed that CBP expression was not altered by downregulation or upregulation of DPP10-AS1 (Fig. 5C). 
Table II. Potential targets of DPP10-AS $1^{\text {a }}$ predicted by StarBase version 2.0.

\begin{tabular}{llccc}
\hline Target gene ID & Pair gene name & Total reads number & Free energy & Align score \\
\hline ENSG00000089123 & TASP1 & 2 & -39.5 & -44.8 \\
ENSG00000105700 & KXD1 & 1 & -39 & 25 \\
ENSG00000113163 & COL4A3BP & 1 & -12.2 & 16 \\
ENSG00000133316 & WDR74 & 1 & -12.2 & 16 \\
ENSG00000222328 & RNU2-2P & 1 & -34 & 16 \\
ENSG00000233876 & GAPDHP68 & 1 & -25.7 & 21.5 \\
Entrez100008588 & RNA18N5 & 1 & 16 \\
\hline
\end{tabular}

${ }^{\mathrm{a}}$ Gene ID, ENSG00000235026.
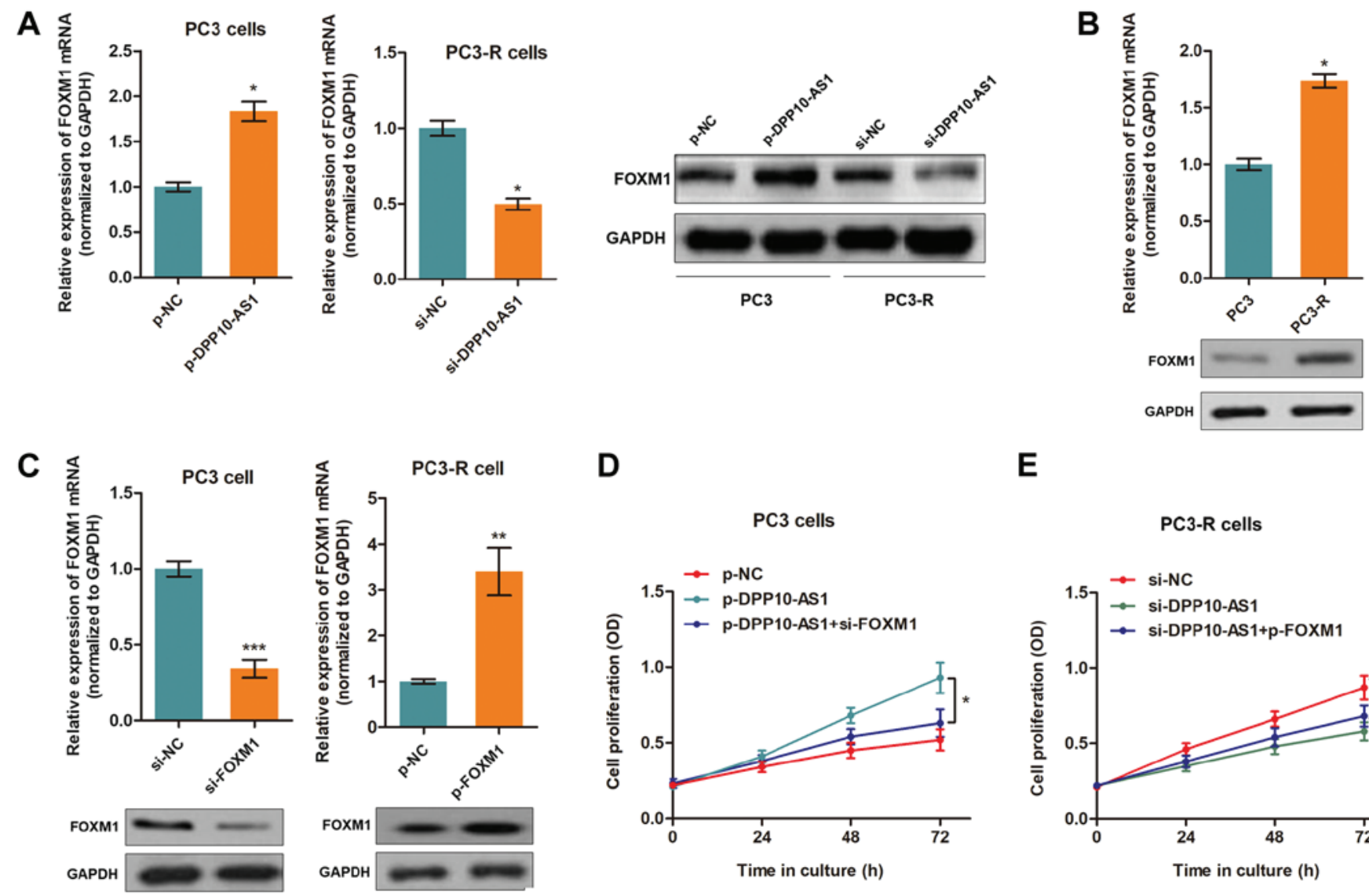

E

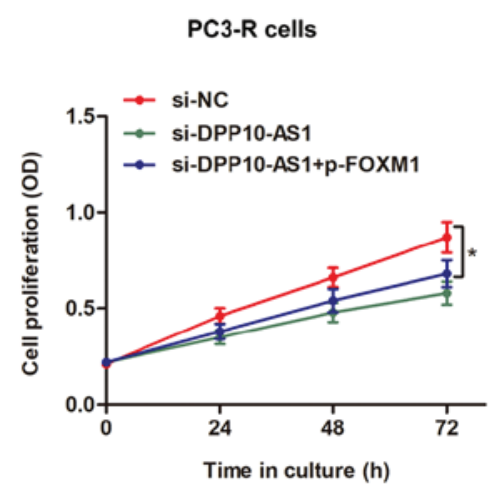

Figure 3. DPP10-AS1 targets FOXM1 in PCa cells. (A) FOXM1 expression was upregulated in the DPP10-AS1 overexpressing PC3 cells. Silencing of DPP10-AS1 resulted in downregulation of PC3-R cells at both the mRNA and protein expression levels. " $\mathrm{P}<0.05$. (B) The mRNA and protein expression levels of FOXM1 were upregulated in PC3-R cells compared with PC3 cells. ${ }^{*}<0.05$. (C) Successful knockdown or overexpression of FOXM1 in cells transfected with a specific siRNA or overexpression vector, respectively. ${ }^{* * *} \mathrm{P}<0.01,{ }^{* * *} \mathrm{P}<0.001$. (D) A CCK8 assay showed that knockdown of FOXM1 partially reversed the DPP10-AS1-induced increase in cell proliferation in PC3 cells, "P<0.05. (E) A CCK8 assay showed that overexpression of FOXM1 abrogated the effects induced by si-DPP10-AS1 in PC3-R cells, "P<0.05. FOXM1, forkhead box M1; PCa, prostate cancer; CCK-8, Cell Counting Kit-8; si, small interfering; $\mathrm{OD}$, optical density; $\mathrm{NC}$, negative control; $\mathrm{p}-$, pcDNA3.1 overexpression vector.

Thus it was hypothesized that DPP10-AS1 may recruit CBP rather than modulate its expression. By performing RIP assays with an anti-CBP antibody, it was shown that DPP10-AS1 was associated with CBP, and enrichment was higher in the PC3-R cells compared with the PC3 cells (Fig. 5E). In addition, CBP was enriched at the FOXM1 promoter region, and dysregulated expression of DPP10-AS1 affected the enrichment of CBP at the FOXM1 promoter (Fig. 5F). Together, these results showed that DPP10-AS1 interacted with CBP, thereby inducing H3K27ac modification in the FOXM1 promoter region.

TMP suppresses tumor growth of $P C a$ in vivo by targeting DPP10-AS1. To identify the suppressive function of TMP during PCa progression, an in vivo nude mouse model bearing a PC3 xenograft was established. PC3 cells stably overexpressing 


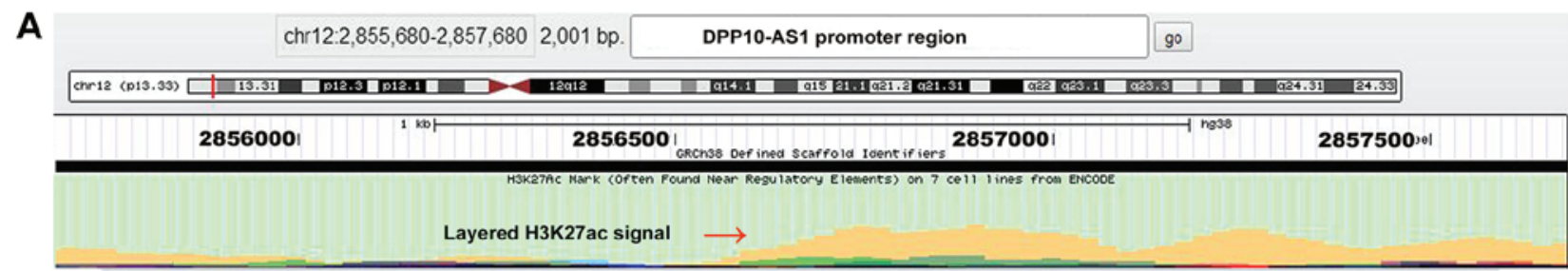
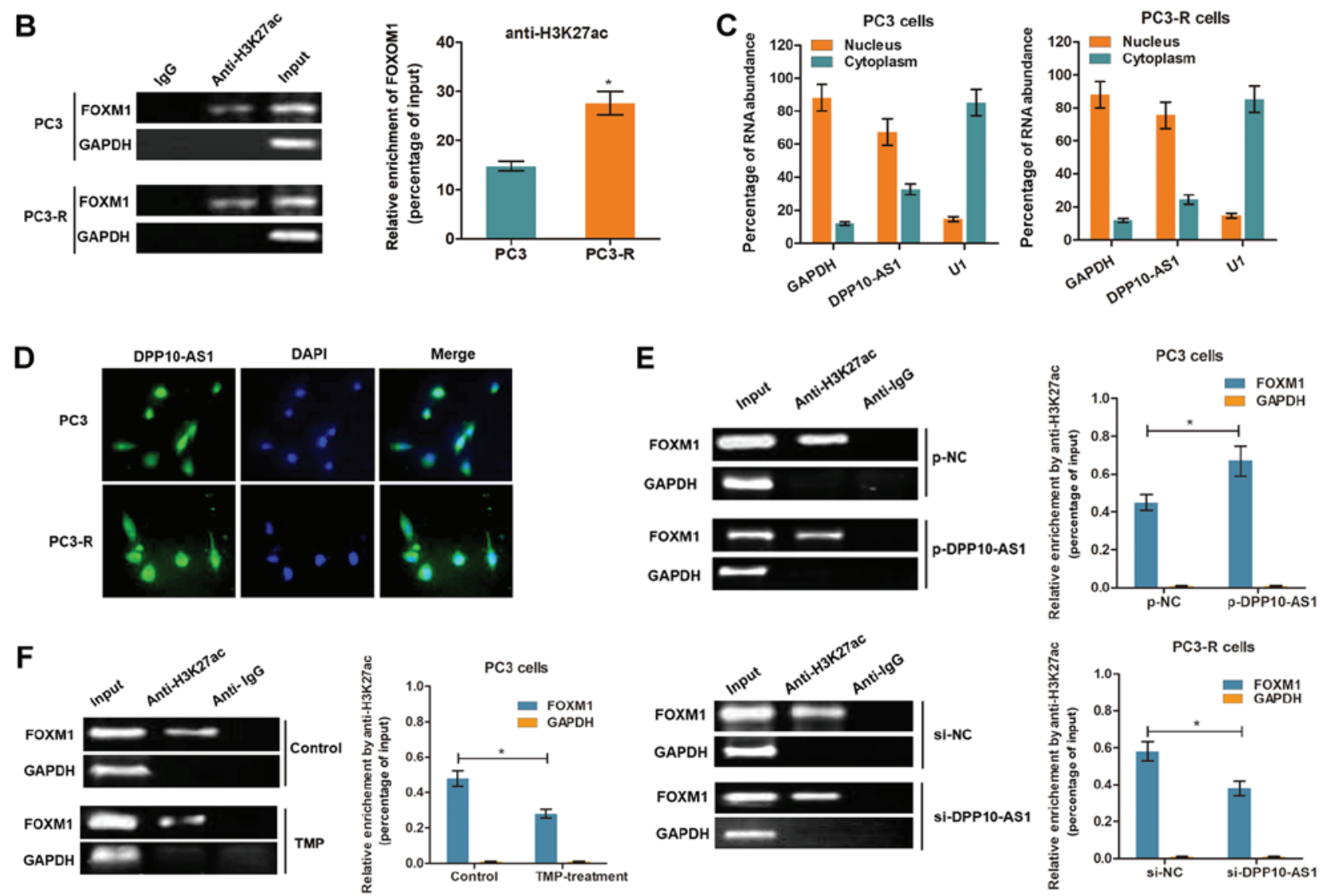

Figure 4. DPP10-AS1 increases the expression of FOXM1 by modifying H3K27 acetylation. (A) Bioinformatics analysis predicted that the promoter region of FOXM1 possessed $\mathrm{H} 3 \mathrm{k} 27 \mathrm{c}$ binding areas. (B) A chromatin immunoprecipitation assay was used to verify the enrichment of $\mathrm{H} 3 \mathrm{~K} 27 \mathrm{ac}$ at the FOXM1 promoter region in PC3 and PC3-R cells. "P<0.05. (C) A nucleocytoplasmic separation-based quantitative PCR assay verified that DPP10-AS1 was primarily distributed in the nucleus of PC3 and PC3-R cells. GAPDH served as the cytoplasmic positive control, and U1 was used as the nuclear positive control. (D) Fluorescence in situ hybridization analysis confirmed the subcellular location of DPP10-AS1 using a specific probe in PC3 and PC3-R cells. (E) DPP10-AS1 positively regulated the enrichment of H3K27ac at FOXM1 promoter regions in both PC3 and PC3-R cells. "P<0.05. (F) TMP treatment decreased the degree of enrichment of H3K27ac at the promoter regions of FOXM1 in PC3 cells. "P<0.05. TMP, tetramethylpyrazine; FOXM1, forkhead box M1; NC, negative control; p-, pcDNA3.1 overexpression vector.

DPP10-AS1 or a negative control vector were injected into the underarm area of mice. Quantitative data showed that TMP treatment significantly reduced tumor growth in Group II vs. I; however, this effect was significantly reversed by co-expression of DPP10-AS1 (Group III vs. II; Fig. 6A).

The expression levels of FOXM1 was determined in tumor tissues using an IHC assay. As shown in Fig. 6B, FOXM1 expression levels were significantly reduced in tumor tissues from mice treated with TMP (Group II vs. I), and this alteration was partially reversed in tissues co-transfected with DPP10-AS1 (Group III vs. II).

\section{Discussion}

Patients with advanced $\mathrm{PCa}$ who develop resistance to hormone therapy have limited therapeutic options in the clinic at present, and therefore, several patients turn to alternative treatments (23). In recent years, the interest in herbal remedies has grown rapidly in the industrialized world, since these drugs are increasingly considered as effective and safe alternatives to synthetic drugs (24-26). In the present study, the effects of TMP on PCa, and the underlying mechanisms were determined. TMP is the standardized unique extract from Ligusticum chuanxiong, and is one of the few well-established plant products. TMP was shown to exhibit anti-cancer effects in PCa by downregulating the expression levels of IncRNA DPP10-AS1. Mechanistically, DPP10-AS1 suppressed FOXM1 expression via histone modification at the FOXM1 promoter region. These data suggest that TMP may serve as a useful therapeutic option for treatment of patients with $\mathrm{PCa}$, and lncRNA DPP10-AS1 may serve as a potential therapeutic target. 
A

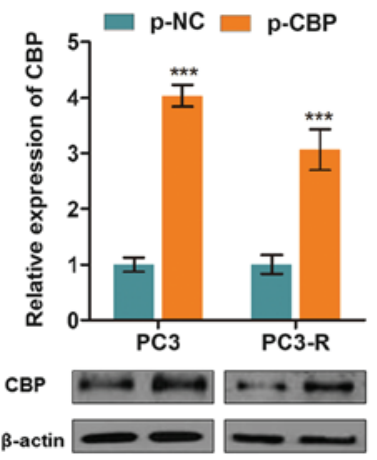

B

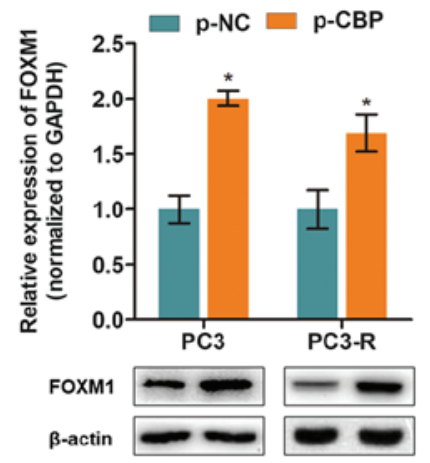

C

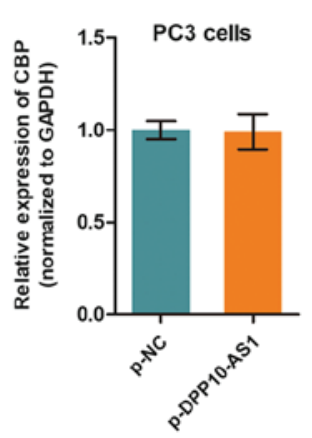

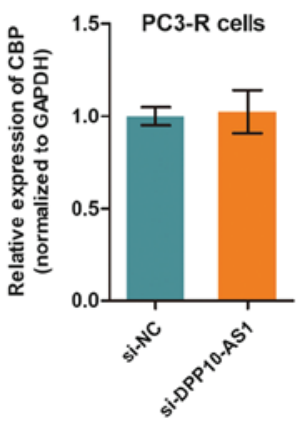

D
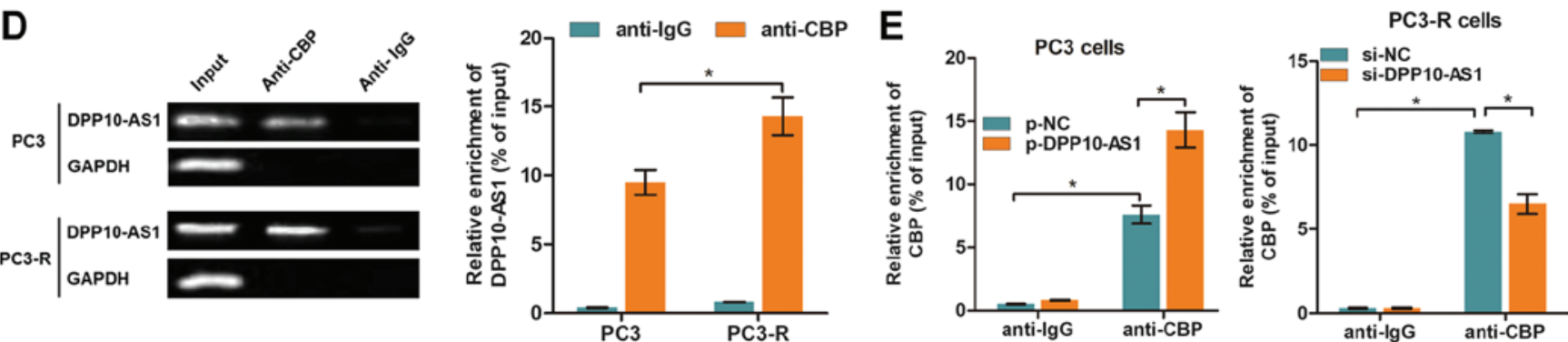

Figure 5. DPP10-AS1 mediates H3K27 acetylation at the promoter region of FOXM1 by binding with CBP. (A) CBP was upregulated by transfection of CBP overexpression vectors in PCa cells, ${ }^{* * *} \mathrm{P}<0.001$. (B) FOXM1 protein expression levels were upregulated by CBP overexpression in PC3 and PC3-R cells. $\mathrm{P}<0.05$. (C) CBP expression was not altered by DPP10-AS1 overexpression in PCa cells. (D) RNA immunoprecipitation assays showed that DPP10-AS1 levels were enriched using a CBP antibody, and the degree of enrichment was significantly higher in the PC3-R cells compared with the PC3 cells. ${ }^{*} \mathrm{P}<0.05$. (E) Chromatin immunoprecipitation-quantitative PCR revealed that DPP10-AS1 influenced the enrichment of CBP at the promoter region of FOXM1. "P<0.05. CBP, CREB binding protein; FOXM1, forkhead box M1; TMP, tetramethylpyrazine; si, small interfering; NC, negative control; p-, pcDNA3.1 overexpression vector.
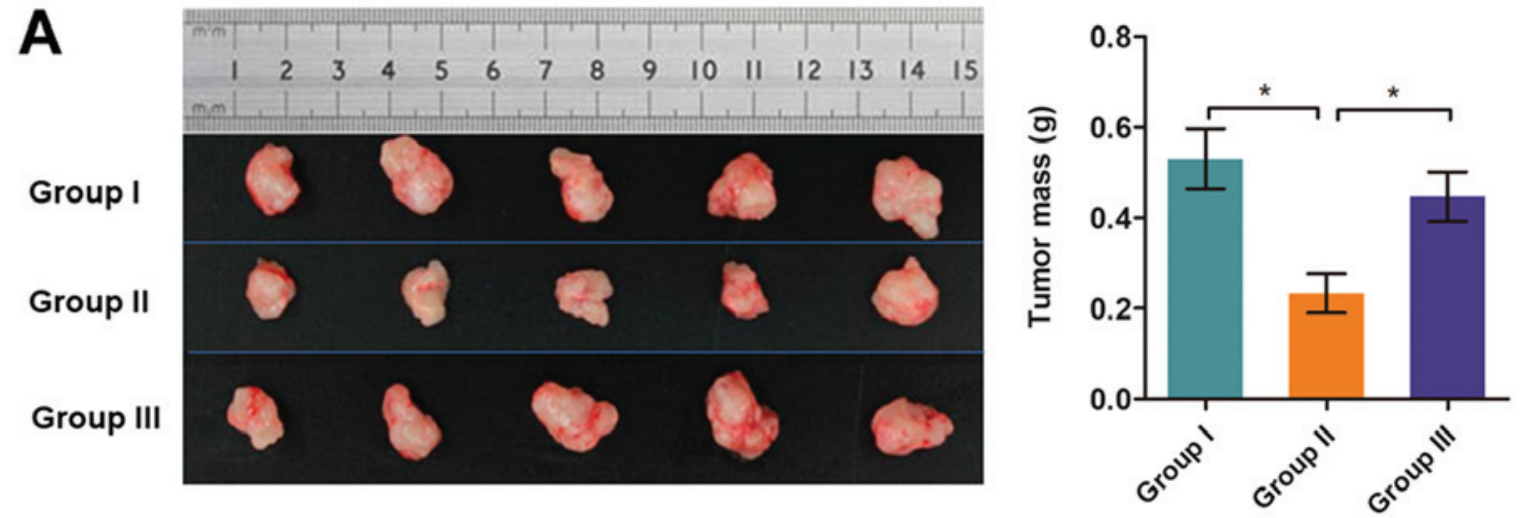

B

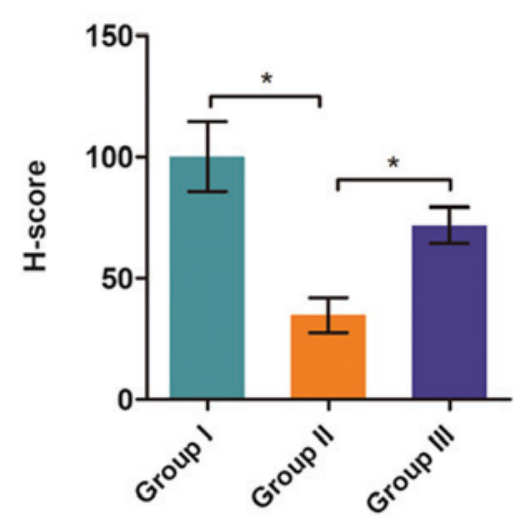

Figure 6. DPP10-AS1 facilitates TMP-induced anti-cancer effects via FOXM1 in vivo. (A) Representative tumors from the xenograft mouse model treated with TMP or PBS (left panel) and tumor mass (right panel). ${ }^{*} \mathrm{P}<0.05$. (B) Immunochemistry analysis of the expression levels of FOXM1 protein in each group. The histogram shows the H-score of each group. "P<0.05. TMP, tetramethylpyrazine; FOXM1, forkhead box M1; Group I, PBS treatment + p-NC; Group II, TMP treatment + p-NC; Group III, TMP treatment + p-DPP10-AS1 
TMP is a major bioactive component of Ligusticum chuanxiong which has been shown to reduce the initiation and progression of cardiovascular diseases (27), and exhibits anti-inflammatory properties in several pathological processes (28). Several studies have also shown that TMP exhibits anti-cancer properties. Cao et al (29) showed that TMP inhibited progression of hepatocellular carcinoma by inducing apoptosis and autophagy. Jia et al (30) demonstrated that TMP treatment suppressed lung cancer growth through disruption of angiogenesis via regulation of the BMP/Smad/Id-1 signaling pathway. Consistently, in the present study, the anti-cancer properties of TMP in PCa were demonstrated. PC3 and DU145 cells exhibit differing sensitivities to TMP, possibly due to the difference of cell membrane permeability and material transport (10). In our previous study, the anti-tumor role of TMP in PCa was shown to be primarily mediated through downregulation of FOXM1. However, the detailed regulatory mechanism were unclear. In the present study, the role of lncRNA DPP10-AS1 in TMP-mediated effects was demonstrated.

Antisense lncRNAs are a group of noncoding genes oriented from protein coding or noncoding loci in the opposite respective direction, and widely participate in the regulation of multiple biological and pathological processes (31). These noncoding antisense transcripts, consistent with other types of IncRNAs, may serve as oncogenes or tumor suppressor genes through upregulation of transcription of specific genes (32). For example, lncRNA ZEB1-AS1 functions as an oncogene in prostate cancer through epigenetic activation of ZEB1 and indirectly regulating downstream molecules (33). LncRNA DPP10-AS1 is localized in the antisense DNA stand of the DPP1O gene, which is strongly associated with asthma susceptibility, possibly through regulating the activities of chemokines and cytokines (34). However, the function of DPP10-AS1 in cancer occurrence and other diseases have not been reported, to the best of our knowledge, and thus the present study is the first to investigate the role of DPP10-AS1 in PCa progression and therapy.

As the essential role of FOXM1 in TMP-induced anti-tumor effects were demonstrated in our previous study, the presence of a functional link between DPP10-AS1 and FOXM1 was assessed in the present study. By overexpressing or knocking-down FOXM1 expression, FOXM1 was verified as a direct target of DPP10-AS1, and was responsible for the functional effects of DPP10-AS1. To determine how DPP10-AS1 affected FOXM1 expression, the epigenetic modifications of FOXM1 in PCa cells were determined, as described previously $(35,36)$. DNA methylation and acetylation are two common epigenetic modifications that are essential in the maintenance of transcription, and closely associated with progression and prognosis of various diseases (37-39). A previous study showed that epigenetic modifications may influence protein coding loci as well as noncoding loci based on a genome-wide sequence analysis (40). Histone acetylation is a common epigenetic modification at gene promoter regions which activate transcription, may induce upregulation of associated transcripts, and thus may be associated with malignant progression (41). For example, Myd88 is activated due to $\mathrm{H} 3 \mathrm{~K} 27 \mathrm{ac}$ modification at the promoter region in hepatocellular carcinoma, and this results in tumor growth and metastasis (42); SNHG14 noncoding RNA activated PABPC1 transcription via the H3K27ac modification at its promoter region, further regulating chemoresistance in breast cancer (43).

Histone acetylation is required for regulating gene transcription by promoting or repressing DNA replication activity $(44,45)$. The role of CBP in DPP10-AS1-mediated histone acetylation was also shown in the present study. $\mathrm{CBP} / \mathrm{p} 300$ was the first discovered mammalian histone acetyltransferase, and belongs to the GCN5-related $\mathrm{N}$-acetyltransferase family (46). CBP-mediated acetylation of $\mathrm{H} 3 \mathrm{~K} 27$ facilitates the transcription of downstream genes (47). CBP has been reported to be involved in the progression of several types of cancer, such as gastric cancer and lung adenocarcinoma; however, the underlying mechanism by which CBP regulates the pathogenesis of various tumors is still unknown. The present provides an alternate perspective, where CBP interaction with noncoding RNAs may serve as the mechanism underlying cancer progression.

In conclusion, the present study verified the anti-tumor effects of TMP in PCa, and further identified the essential role of the DPP10-AS1/CBP/FOXM1 regulatory pathway in TMP-induced suppression of PCa progression. These data suggest the potential significance of DPP10-AS1 as a promising therapeutic target and predictive indicator of $\mathrm{PCa}$ progression.

\section{Acknowledgements}

Not applicable.

\section{Funding}

The present study was supported by The Science Foundation of Beijing.

\section{Availability of data and materials}

The datasets used and/or analyzed during the present study are available from the corresponding author on reasonable request.

\section{Authors' contributions}

YZ, ZJ and WY designed the study and performed the experiments. ZZ, HL and XU analyzed the data. All authors read and approved the final manuscript.

\section{Ethics approval and consent to participate}

The study protocol was approved by the Research Ethics Committee of Peking union Medical College Hospital.

\section{Patient consent for publication}

Not applicable.

\section{Competing interests}

The authors declare that they have no competing interests. 


\section{References}

1. Schatten H: Brief overview of prostate cancer statistics, grading, diagnosis and treatment strategies. Adv Exp Med Biol 1095: 1-14, 2018.

2. Horiguchi M, Uno H and Wei LJ: Evaluating noninferiority with clinically interpretable statistics for the PROSELICA study to assess treatment efficacy of a reduced dose of cabazitaxel for treating metastatic prostate cancer. J Clin Oncol 36: 825-826, 2018.

3. Lunardi A, Ala U, Epping MT, Salmena L, Clohessy JG, Webster KA, Wang G, Mazzucchelli R, Bianconi M, Stack EC, et al: A co-clinical approach identifies mechanisms and potential therapies for androgen deprivation resistance in prostate cancer. Nat Genet 45: 747-755, 2013.

4. Yu N, Zhang Z, Chen P, Zhong Y, Cai X, Hu H, Yang Y, Zhang J, Li K, Ge J, et al: Tetramethylpyrazine (TMP), an active ingredient of Chinese herb medicine chuanxiong, attenuates the degeneration of trabecular meshwork through SDF-1/CXCR4 axis. PLoS One 10: e0133055, 2015.

5. Zeng Z, Zhu W, Zhou X, Jin Z, Liu H, Chen X, Pan J, Demura H, Naruse $M$ and Shi Y: Tetramethylpyrazine, a Chinese drug, blocks coronary vasoconstriction by endothelin- 1 and decreases plasma endothelin-1 levels in experimental animals. J Cardiovase Pharmacol 31 (Suppl 1): S313-S316, 1998.

6. Huang HH, Liu FB, Ruan Z, Zheng J, Su YJ and Wang J: Tetramethylpyrazine (TMPZ) triggers S-phase arrest and mitochondria-dependent apoptosis in lung cancer cells. Neoplasma 65: 367-375, 2018.

7. Wang S, Lei $\mathrm{T}$ and Zhang M: The reversal effect and its mechanisms of tetramethylpyrazine on multidrug resistance in human bladder cancer. PLoS One 11: e0157759, 2016.

8. Zhang Y, Liu X, Zuo T, Liu Y and Zhang JH: Tetramethylpyrazine reverses multidrug resistance in breast cancer cells through regulating the expression and function of P-glycoprotein. Med Oncol 29: 534-538, 2012.

9. Yi B, Liu D, He M,LiQ, Liu T and Shao J: Role of the ROS/AMPK signaling pathway in tetramethylpyrazine-induced apoptosis in gastric cancer cells. Oncol Lett 6: 583-589, 2013.

10. Zhou Y,JiZ, Yan W,Zhou Z,LiH and Xiao Y: Tetramethylpyrazine inhibits prostate cancer progression by downregulation of forkhead box M1. Oncol Rep 38: 837-842, 2017.

11. Ponting CP, Oliver PL and Reik W: Evolution and functions of long noncoding RNAs. Cell 136: 629-641, 2009.

12. Kopp F and Mendell JT: Functional classification and experimental dissection of long noncoding RNAs. Cell 172: 393-407, 2018.

13. Rafiee A, Riazi-Rad F, Havaskary M and Nuri F: Long noncoding RNAs: Regulation, function and cancer. Biotechnol Genet Eng Rev 34: 153-180, 2018.

14. Josipovic I, Pflüger B, Fork C, Vasconez AE, Oo JA, Hitzel J, Seredinski S, Gamen E, Heringdorf DMZ, Chen W, et al: Long noncoding RNA LISPR1 is required for S1P signaling and endothelial cell function. J Mol Cell Cardiol 116: 57-68, 2018.

15. Magistri M, Faghihi MA, St Laurent G III and Wahlestedt C: Regulation of chromatin structure by long noncoding RNAs: Focus on natural antisense transcripts. Trends Genet 28: 389-396, 2012.

16. Li W, Sun M, Zang C, Ma P, He J, Zhang M, Huang Z, Ding Y and Shu Y: Upregulated long non-coding RNA AGAP2-AS1 represses LATS2 and KLF2 expression through interacting with EZH2 and LSD1 in non-small-cell lung cancer cells. Cell Death Dis 7: e2225, 2016.

17. Chen C, Feng Y and Wang X: LncRNA ZEB1-AS1 expression in cancer prognosis: Review and meta-analysis. Clin Chim Acta 484: 265-271, 2018

18. Livak KJ and Schmittgen TD: Analysis of relative gene expression data using real-time quantitative PCR and the 2(-Delta Delta C(T)) method. Methods 25: 402-408, 2001.

19. Kent WJ, Sugnet CW, Furey TS, Roskin KM, Pringle TH, Zahler AM and Haussler D: The human genome browser at UCSC. Genome Res 12: 996-1006, 2002.

20. Li JH, Liu S, Zhou H, Qu LH and Yang JH: starBase v2.0: Decoding miRNA-ceRNA, miRNA-ncRNA and protein-RNA interaction networks from large-scale CLIP-Seq data. Nucleic Acids Res 42: D92-D97, 2014.

21. Lv C, Zhao G, Sun X, Wang P, Xie N, Luo J and Tong T: Acetylation of FOXM1 is essential for its transactivation and tumor growth stimulation. Oncotarget 7: 60366-60382, 2016.
22. Wang L, Tang Y, Cole PA and Marmorstein R: Structure and chemistry of the p300/CBP and Rtt109 histone acetyltransferases: Implications for histone acetyltransferase evolution and function. Curr Opin Struct Biol 18: 741-747, 2008.

23. Curry EA III and Sweeney CJ: Resistance to luteinizing hormone releasing hormone agonist therapy for metastatic prostate cancer. J Urol 168: 193, 2002.

24. McCarty MF: Current prospects for controlling cancer growth with non-cytotoxic agents-nutrients, phytochemicals, herbal extracts, and available drugs. Med Hypotheses 56: 137-154, 2001.

25. Muhamad N, Plengsuriyakarn $\mathrm{T}$ and Na-Bangchang K: Application of active targeting nanoparticle delivery system for chemotherapeutic drugs and traditional/herbal medicines in cancer therapy: A systematic review. Int J Nanomedicine 13: 3921-3935, 2018

26. Vinayak M: Molecular action of herbal antioxidants in regulation of cancer growth: Scope for novel anticancer drugs. Nutr Cancer 70: 1199-1209, 2018.

27. Guo M, Liu Y and Shi D: Cardiovascular actions and therapeutic potential of tetramethylpyrazine (Active Component Isolated from Rhizoma Chuanxiong): Roles and mechanisms. Biomed Res Int 2016: 2430329, 2016.

28. Hu JZ, Huang JH, Xiao ZM, Li JH, Li XM and Lu HB Tetramethylpyrazine accelerates the function recovery of traumatic spinal cord in rat model by attenuating inflammation. J Neurol Sci 324: 94-99, 2013.

29. Cao J, Miao Q, Miao S, Bi L, Zhang S, Yang Q, Zhou X, Zhang M, Xie Y, Zhang J and Wang S: Tetramethylpyrazine (TMP) exerts antitumor effects by inducing apoptosis and autophagy in hepatocellular carcinoma. Int Immunopharmacol 26: 212-220, 2015.

30. Jia Y, Wang Z, Zang A, Jiao S, Chen S and Fu Y Tetramethylpyrazine inhibits tumor growth of lung cancer through disrupting angiogenesis via BMP/Smad/Id-1 signaling. Int J Oncol 48: 2079-2086, 2016.

31. Jadaliha M, Gholamalamdari O, Tang W, Zhang Y, Petracovici A, Hao Q, Tariq A, Kim TG, Holton SE, Singh DK, et al: A natural antisense lncRNA controls breast cancer progression by promoting tumor suppressor gene mRNA stability. PLoS Genet 14: e1007802, 2018

32. Pian L, Wen X, Kang L, Li Z, Nie Y, Du Z, Yu D, Zhou L, Jia L, Chen N, et al: Targeting the IGF1R pathway in breast cancer using antisense lncRNA-mediated promoter cis competition. Mol Ther Nucleic Acids 12: 105-117, 2018

33. Su W, Xu M, Chen X, Chen N, Gong J, Nie L, Li L, Li X, Zhang M and Zhou Q: Long noncoding RNA ZEB1-AS1 epigenetically regulates the expressions of ZEB1 and downstream molecules in prostate cancer. Mol Cancer 16: 142, 2017.

34. Belau F, Metzner K, Christ T, Ravens U, Schaefer M, Künzel S, Li W, Wettwer E, Dobrev D, El-Armouche A and Kämmerer S: DPP10 is a new regulator of Nav1.5 channels in human heart. Int J Cardiol 284: 68-73, 2019.

35. Pérez-Peña J, Győrffy B, Amir E, Pandiella A and Ocaña A: Epigenetic modulation of FOXM1-gene interacting network by BET inhibitors in breast cancer. Breast Cancer Res Treat 172: 725-732, 2018

36. Zhou Z, Chen H, Xie R, Wang H, Li S, Xu Q, Xu N, Cheng Q, Qian Y, Huang R, et al: Epigenetically modulated FOXM1 suppresses dendritic cell maturation in pancreatic cancer and colon cancer. Mol Oncol 13: 873-893, 2019.

37. Shi H, Wei SH, Leu YW, Rahmatpanah F, Liu JC, Yan PS, Nephew KP and Huang TH: Triple analysis of the cancer epigenome: An integrated microarray system for assessing gene expression, DNA methylation, and histone acetylation. Cancer Res 63: 2164-2171, 2003.

38. Tang RZ, Zhu JJ, Yang FF, Zhang YP, Xie SA, Liu YF, Yao WJ, Pang W, Han LL, Kong W, et al: DNA methyltransferase 1 and Krüppel-like factor 4 axis regulates macrophage inflammation and atherosclerosis. J Mol Cell Cardiol 128: 11-24, 2019.

39. Liu L, He X, Zhao M, Yang S, Wang S, Yu X, Liu J and Zang W: Regulation of DNA methylation and 2-OG/TET signaling by choline alleviated cardiac hypertrophy in spontaneously hypertensive rats. J Mol Cell Cardiol 128: 26-37, 2019.

40. Wan G, Hu X, Liu Y, Han C, Sood AK, Calin GA, Zhang X and $\mathrm{Lu} \mathrm{X}$ : A novel non-coding RNA lncRNA-JADE connects DNA damage signalling to histone $\mathrm{H} 4$ acetylation. EMBO J 32: 2833-2847, 2013.

41. Li D, Bi FF, Cao JM, Cao C, Liu B and Yang Q: Regulation of DNA methyltransferase 1 transcription in BRCA1-mutated breast cancer: A novel crosstalk between E2F1 motif hypermethylation and loss of histone H3 lysine 9 acetylation. Mol Cancer 13: 26, 2014. 
42. Xu X, Yin Y, Tang J, Xie Y, Han Z, Zhang X, Liu Q, Qin X, Huang $X$ and Sun B: Long non-coding RNA Myd88 promotes growth and metastasis in hepatocellular carcinoma via regulating Myd88 expression through H3K27 modification. Cell Death Dis 8: e3124, 2017.

43. Dong H, Wang W, Mo S, Liu Q, Chen X, Chen R, Zhang Y, Zou K, Ye M, He X, et al: Long non-coding RNA SNHG14 induces trastuzumab resistance of breast cancer via regulating PABPC1 expression through H3K27 acetylation. J Cell Mol Med 22: 4935-4947, 2018.

44. Das C, Lucia MS, Hansen KC and Tyler JK: CBP/p300-mediated acetylation of histone H3 on lysine 56. Nature 459: 113-117, 2009.
45. Ghosh TK, Aparicio-Sánchez JJ, Buxton S, Ketley A, Mohamed T, Rutland CS, Loughna S and Brook JD: Acetylation of TBX5 by KAT2B and KAT2A regulates heart and limb development. J Mol Cell Cardiol 114: 185-198, 2018.

46. Wang YM, Gu ML, Meng FS, Jiao WR, Zhou XX, Yao HP and Ji F: Histone acetyltransferase p300/CBP inhibitor C646 blocks the survival and invasion pathways of gastric cancer cell lines. Int J Oncol 51: 1860-1868, 2017.

47. Kouzarides T: SnapShot: Histone-modifying enzymes. Cell 131: 822, 2007. 